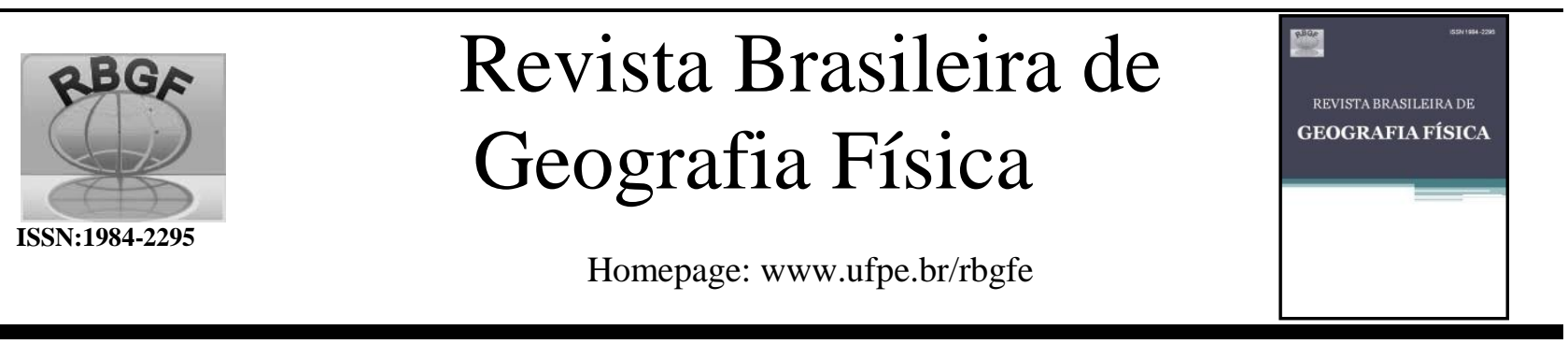

\title{
Espacialização dos parâmetros de equações de chuvas intensas para a Região Metropolitana do Recife
}

\author{
Ana Emília Carvalho de Gusmão da Cunha Rabelo ${ }^{1}$, Larissa Virgínia da Silva Ribas ${ }^{2}$, Artur Paiva
} Coutinho $^{3}$, Alfredo Ribeiro Neto ${ }^{4}$, Antonio Celso Dantas Antonino ${ }^{5}$

${ }^{1}$ Estudante do Programa de Pós-Graduação em Tecnologias Energéticas e Nucleares, da Universidade Federal de Pernambuco-UFPE. Av. Luiz Freire, 1000. Cidade Universitária, Recife-PE, Brasil. CEP: 50740-545. Autor correspondente: E-mail: anaerabelo@gmail.com; ${ }^{2}$ Estudante do Programa de Pós-Graduação em Engenharia Civil. E-mail:larissa.vribas @ hotmail.com; ${ }^{3}$ Prof. do Núcleo de Tecnologia do Centro Acadêmico do Agreste da UFPE. E-mail: arthur.coutinho@yahoo.com.br; ${ }^{4}$ Prof. do Departamento de Engenharia Civil e Ambiental da UFPE, Centro de Tecnologia e Geociências. E-mail: ribeiront@gmail.com; ${ }^{5}$ Prof. do Departamento de Energia Nuclear da UFPE. E-mail: acdantonino@gmail.com.

Artigo recebido em 13/02/2017 e aceito em 23/05/2018

\section{R E S U M O}

As equações de chuvas intensas são um dos meios mais utilizados para obtenção das estimativas de vazões de projeto aplicadas no dimensionamento de obras hidráulicas. No entanto, devido à escassez de dados pluviométricos em determinadas regiões do país, a determinação dessas equações fica por vezes comprometida, dificultando a determinação de chuvas de projeto. O objetivo principal deste estudo é utilizar um método de interpolação matemática, inverso do peso da distância - IDW, para espacialização dos parâmetros de equações de chuvas intensas para a Região Metropolitana do Recife (RMR) e Município de Goiana, a partir de 17 estações pluviométricas representativas e estrategicamente localizadas. Como resultados são apresentados mapas de distribuição espacial para cada um dos parâmetros de equações de chuvas intensas da RMR, possibilitando o cálculo de uma precipitação associada a uma duração e a um tempo de retorno para qualquer local da RMR. Foi ainda realizada a verificação do erro entre as intensidades calculadas e parametrizadas para validação do estudo. Sendo também representados mapas de distribuição ao desconsiderar a Estação de Camaragibe, para deste modo realizar a avaliação dos erros percentuais envolvendo tal análise, onde, neste caso, os mesmos se apresentaram baixos, variando de 0,05 até 5,43\%.

Palavras-chave: Equações i-d-f, Interpolação por IDW, Precipitação, Variabilidade Espacial.

\section{Spatialization of intense rainfall equations parameters for the Metropolitan Region of Recife}

\section{A B S T R A C T}

Intense precipitation equations are one of the main ways to obtain the design flow estimative applied to the design of hydraulic structures. However, due to the shortage rainfall data in certain regions of the country, the determination of these equations is sometimes compromised, what difficulties the determination of design rains. The main aim of this study is to use a method of mathematical interpolation, Inverse Distance Weighting - IDW to do the spatialization from intense rainfall parameters for the Metropolitan Region of Recife (RMR) and the city of Goiana. Those will be made through 17 representative rainfall stations, which are strategically located. As results are shown maps of spatial distribution, for each parameter of intense rainfalls in the RMR. Therefore, enabling the calculation of an associated rainfall with duration and time of return on any location of the RMR. It was also conducted the verification of the error between the parameterized calculated intensities the validation of the study. It was also represented distribution maps when Camaragibe station was disregarded, thereby to conduct the evaluation of the percentage errors involving such analysis, which, in this case showed to be low, ranging from 0.05 to $5.43 \%$. 
Keywords: i-d-f Equations, IDW Interpolation, Precipitation, Spatial variability.

\section{Introdução}

Os números de eventos de cheias, inundações e deslizamentos de encostas tem aumentado nas áreas urbanas, causando danos materiais e mortes. Além de deixar pessoas desabrigadas e famílias desestruturadas, facilita também a proliferação de doenças de veiculação hídrica, tais como: leptospirose, hepatite, cólera, giardíase, entre outras. Isto se deve à urbanização desordenada e sem controle, a qual aumenta a área de superfícies impermeáveis, desencadeando problemas no sistema de drenagem urbana e de captação de águas pluviais, assim como mudanças no ciclo hidrológico do ambiente urbano.

A ocupação de área de mangues, alagados, leito dos rios, morros e áreas de Mata Atlântica, contribuem com a mudança do cenário urbano e, consequentemente, com a mudança de percurso e quantidade de água que escoa superficialmente. Desse modo, tem-se um aumento da velocidade e volume do escoamento pluvial e, em contrapartida, uma diminuição da infiltração, que em conjunto com os dispositivos de drenagem urbana ineficazes e subestimados, leva as cidades a estados de emergência e calamidade. Assim, como a maioria das grandes cidades brasileiras, Recife e sua Região Metropolitana vêm sofrendo com inúmeros problemas de alagamentos, transbordamentos de córregos e rios e deslizamentos de morros, alarmando para um cenário preocupante, principalmente para a população de baixa renda, a qual, em sua maioria, está alocada em regiões vulneráveis ao acontecimento desses tipos de desastres.

Diante da situação de risco a qual a população está sujeita, mediante a ocorrência de tais eventos, é de estrema necessidade o conhecimento da variabilidade espacial das variáveis climatológicas de uma região. Essas irão auxiliar na obtenção de dados de planejamento territorial e gestão de riscos. A espacialização dos parâmetros de equações de chuvas intensas permitiria a obtenção das estimativas de vazões de projeto aplicadas no dimensionamento de obras hidráulicas, na drenagem urbana e na drenagem agrícola, em regiões que não possuem dados suficientes para obtenção de uma i-d-f própria. Assim, a determinação da intensidade das precipitações intensas, o mais precisa possível, é extremamente importante para que um projeto seja viável tecnicamente e economicamente (Mello et al., 2003).

No meio técnico-científico brasileiro, os entraves existentes para a determinação dos hietogramas de precipitações máximas associadas a uma duração e a um tempo de retorno, necessários para os projetos hidrológicos, são a escassez de dados pluviográficos, a pobre distribuição de postos da rede pluviográfica, além do pequeno período de observações disponível nas séries históricas de precipitações. Nesse contexto, é fundamental a aplicação de metodologias simplificadas de desagregação da chuva diária, valorizando a rede pluviométrica existente em maior número para a estimativa das precipitações de projeto.

Pernambuco, assim como a maioria dos estados brasileiros, conta com uma ampla quantidade de estudos de chuvas intensas (Silva et al., 2012; Albuquerque et al., 2013; Coutinho et al., 2013 ; Coutinho et al., 2013b; Silva e Araújo, 2013), deste modo, o estado dispõe de boas séries históricas de dados pluviométricos, que permitem o uso de métodos simplificadores para determinar a i-d-f. Isto possibilita o uso de metodologias de desagregação de chuvas, tais como o método das relações de durações e o de Bell (1969), o qual foi aplicado neste artigo para obtenção dos parâmetros da i-d-f por meio das máximas chuvas anuais.

Os métodos de interpolação matemática possibilitam uma estimativa do valor de um atributo em locais não amostrados, a partir de locais amostrados pontuais na mesma área, nesse caso, os valores dos parâmetros das equações de chuvas intensas.

Diante da disponibilidade de dados históricos das estações pluviométricas da Região Metropolitana do Recife (RMR), através da Agência Nacional de Águas (ANA), foi utilizada a metodologia de desagregação de chuvas, o método de Bell (1969), para obtenção dos parâmetros das IDF's por meio das máximas chuvas anuais para 17 localidades espaçadas ao longo de toda a região em estudo. Utilizou-se o método de ponderação pelo inverso da distância - IDW para estimar os parâmetros de chuvas intensas através da regressão múltipla não-linear de séries históricas de dados de chuvas máximas.

Cecílio e Pruski (2003) utilizaram o método do inverso da potência da distância para comparar diferentes formas de interpolação dos parâmetros da equação de chuvas intensas. Para tal, eles consideraram as informações existentes de 171 localidades do Estado de Minas Gerais, comparando 625 combinações diferentes entres os parâmetros de chuvas intensas. E verificaram então uma tendência de superestimativa da intensidade de precipitação em todas as combinações. 
Em estudo realizado na França, Tao et al. (2009) analisaram algumas séries de precipitação anuais, mensais, diárias e de seis minutos. Tal análise foi realizada para 30 localidades da grande região de Lyon. Para tanto, foram aplicados os métodos IDW, Spline e Kriging. Eles puderam concluir que tanto os métodos IDW quanto Kriging resultaram em bons valores de saída para a interpolação de valores de precipitações anuais. Porém, o método de Kriging apresentou um resultado levemente melhor que o IDW. Contudo, para análises da precipitação mensal, o IDW se mostrou como sendo o melhor a ser aplicado.

Garcia et al. (2011) determinaram as equações i-d-f para três estações meteorológicas da região centro sul do Estado de Mato Grosso. Eles puderam verificar que a distribuição de Gumbel mostrou-se adequada na estimativa dos valores de intensidade máxima média a nível de significância de 5\%, pelo teste de Kolmogorov-Smirnov. Além disso, os parâmetros das equações intensidadeduração-frequência apresentaram alta variabilidade independentemente do método. $\mathrm{O}$ método da Desagregação não só apresentou melhor desempenho global (Erro médio relativo $(E R M)=$ $27 \%)$, em comparação ao de Bell (ERM $=31 \%)$, como mostrou melhor desempenho em estações com séries mais longas caracterizando-se, assim, como mais sensível ao tamanho da série, em relação ao método de Bell (1969).

O Estado de Goiás e o Distrito Federal também são locais onde estudos foram desenvolvidos no tocante à obtenção das relações i-d-f através do emprego da desagregação da chuva de um dia. Em análise realizada por Oliveira et al. (2007), a distribuição de Gumbel se mostrou adequada para todas as localidades a um nível de significância de $1 \%$ pelo teste de KolmogorovSmirnov. Eles estimaram os parâmetros das relações i-d-f para 73 localizações, as quais apresentaram um coeficiente de determinação acima de $95 \%$, classificado como um bom ajuste. As equações de regressão apresentaram desvios relativos médios entre $-1,6 \%$ e $43,9 \%$ e um bom ajuste de dados, onde os $\mathrm{r}^{2}$ mostraram valores maiores que $99 \%$.

Em estudo realizado por Oliveira et al. (2008), também no estado Goiás, verificou-se a aderência da distribuição de Gumbel em um nível de significância de 5\% pelo teste de KolmogorovSmirnov para as localidades avaliadas com um bom ajuste do método de Bell. Sendo identificado como uma alternativa quando se dispõe de séries históricas com poucos anos de observação e se pretende obter alturas de chuvas intensas.
No estado de Pernambuco e, principalmente, na Região Metropolitana do Recife, inexistem trabalhos relacionados ao estudo da variabilidade espacial dos parâmetros de chuvas intensas. A existência de dados pluviográficos na RMR ainda é escassa. Nesse caso, faz-se necessária a aplicação de métodos de desagregação de chuva, isto é, métodos que transformam uma informação de precipitação pluviométrica com duração diária em precipitações com durações menores. Estudos como os de Taborga (1974) e CETESB (1986) para o método das relações entre durações são exemplos de métodos de desagregação de chuvas para a realidade brasileira.

Taborga (1974) desenvolveu oito isozonas de igual relação para o Brasil, para diferentes tempos de retorno. Nesse estudo foi possível realizar a conversão da chuva de 1 dia para uma chuva de 24 horas e de uma chuva com duração de seis minutos para uma chuva com duração de 24 horas. O estudo de Taborga (1974) é uma das primeiras tentativas de impor uma variabilidade espacial, em grande escala, das precipitações associadas a um tempo de retorno e duração para o território brasileiro.

CETESB (1986) fornece uma tabela que permite calcular as razões entre as precipitações de 24 h, 12 h, 10 h, 8 h, 6 h, 1 h, 30 min, 25 min, 20 min, $15 \mathrm{~min}, 10 \mathrm{~min}$ e $5 \mathrm{~min}$. Essa tabela pode ser obtida para diferentes tempos de retorno.

Nesse contexto, este estudo objetivou utilizar o método matemático de ponderação pelo inverso da distância, usando-se o quadrado como expoente da distância, no tocante à interpolação dos parâmetros das equações de chuvas intensas. Assim, foi possível espacializar as equações na Região Metropolitana do Recife e no município de Goiana, em locais que não apresentam tais informações.

\section{Material e métodos}

A RMR se encontra localizada no Nordeste do Brasil, costeando o Oceano Atlântico e englobando uma área total de $2.768 \mathrm{~km}^{2}$. Esta é formada, atualmente, por 14 municípios: Abreu e Lima, Araçoiaba, Camaragibe, Cabo de Santo Agostinho, Igarassu, Ilha de Itamaracá, Ipojuca, Itapissuma, Jaboatão dos Guararapes, Moreno, Olinda, Paulista, Recife e São Lourenço da Mata, somando uma população de 3.690 .485 habitantes. Estes representam cerca de $51 \%$ da população urbana e apenas $5,81 \%$ da população rural do Estado de Pernambuco (IBGE, 2010). Ainda, de acordo com os estudos do IBGE (2010), a RMR é a $5^{\circ}$ região mais populosa do Brasil, onde se 
concentram as maiores atividades urbanas do Estado, nos quais a grande maioria situa-se no polo central, Recife.

O clima nesta região é caracterizado como quente e tropical úmido, apresentando uma baixa variação sazonal em sua temperatura e média anual de $25^{\circ} \mathrm{C}$. Quanto à precipitação média, essa varia de $2.200 \mathrm{~mm} / \mathrm{ano}$, nas áreas mais litorâneas, até $1.200 \mathrm{~mm} / \mathrm{ano}$ a oeste de São Lourenço da Mata. Desse modo, é possível verificar duas estações bem definidas, uma chuvosa, que vai de março a agosto e outra seca, de setembro a fevereiro (APAC, 2015; CPRM, 2003; Coutinho et al., 2016).

Para o município de Recife, a precipitação pluviométrica no tocante à série histórica de precipitações da estação várzea do APAC, para o período de 1994 a 2010, apresenta uma média anual de 2.173,8 mm, com mínima de 1.255,4 mm e máxima de 3.482,0 mm anuais. Durante o ano, os meses de abril, maio, junho e julho são os que apresentam os maiores totais pluviométricos mensais (Figura 1).

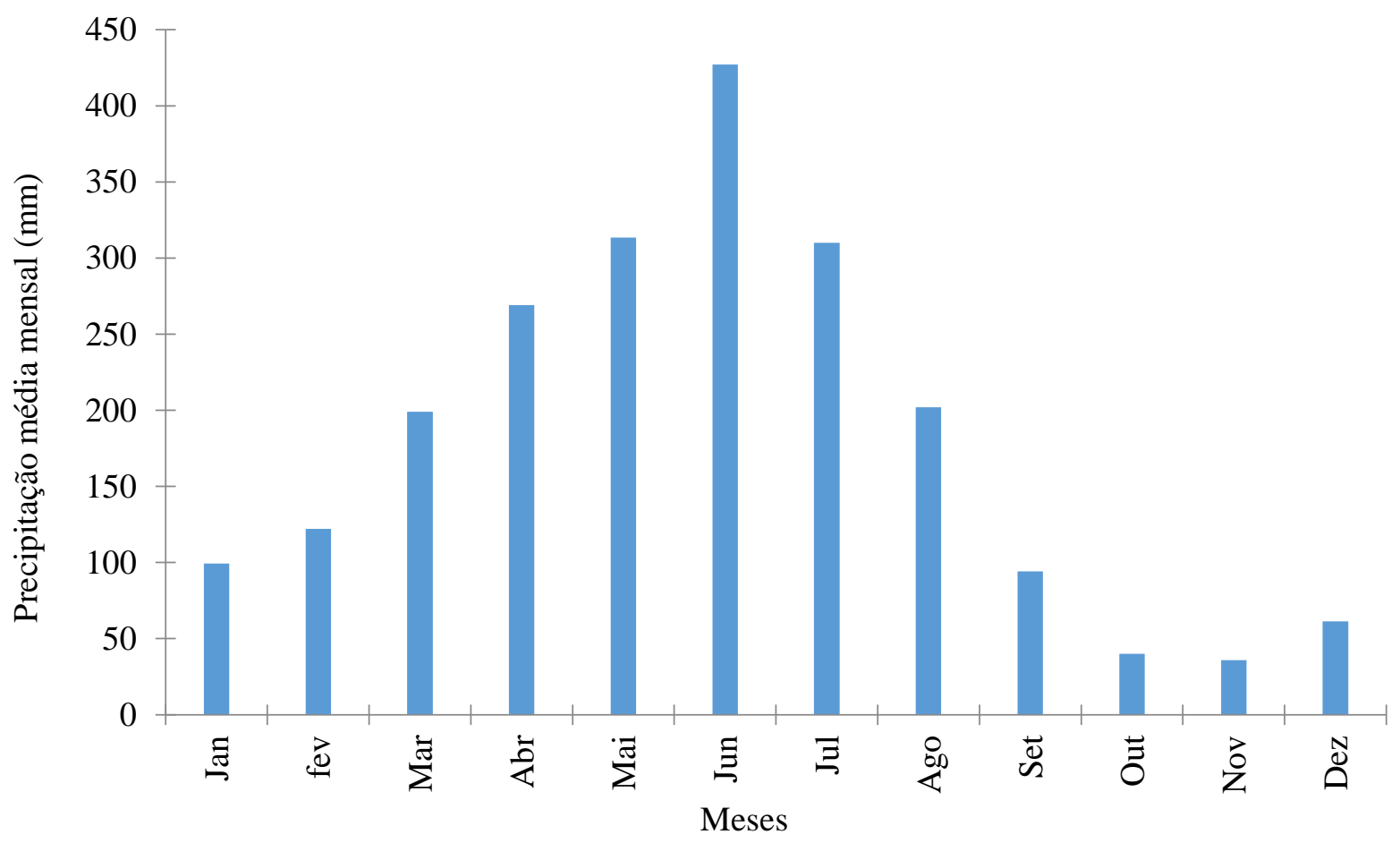

Figura 1- Precipitação média mensal para a cidade do Recife no período 1994-2010. Fonte: APAC/LAMEPE (2015).

Além disso, segundo Cabral e Montenegro (2004), a evaporação anual medida em tanques evaporimétricos na região atinge $1.234,4 \mathrm{~mm}$, com valor máximo em dezembro (146,3 mm), com uma média mensal de $110,3 \mathrm{~mm}$. A umidade relativa do ar possui valor médio mensal de $79,8 \%$, refletindo as proximidades da região com o oceano, o qual libera massas de ar úmido que são trazidas da região da costa do continente.

A principal forma de caracterização de chuvas intensas é por meio da equação de intensidade, duração e frequência da precipitação. Essas equações são, normalmente, representadas da seguinte forma:

$$
i_{m}=\frac{\mathrm{a} \cdot T^{b}}{(t+c)^{d}}
$$

onde: $i_{m}$ - intensidade máxima média de precipitação $(\mathrm{mm} / \mathrm{h}), \mathrm{T}$ - período de retorno, em anos, $\mathrm{t}$ - duração da precipitação, em minutos, a, b, c, d - parâmetros relativos ao local (estação pluviométrica).

A RMR dispõe apenas de 2 equações de chuva, relativas aos postos de Olinda e do Curado, elaboradas, respectivamente, pelas consultoras EMCIBRA e Aquaplan, nas décadas de 1970 e 1980, com dados históricos entre 1926 e 1977.

Diante disso, inicialmente foi realizado um levantamento da quantidade de postos pluviométricos situados nos municípios da RMR que possuem séries históricas pluviais com mais de 15 anos de dados. As séries históricas foram obtidas na base de dados da Agência Nacional de Águas (ANA) e através do website Hidroweb. Os dados pluviométricos foram analisados e tratados 
para correção de falhas, análise de consistência e remoção de erros grosseiros, definindo-se um total de 17 estações viáveis a serem trabalhadas neste artigo. Na Tabela 1 são apresentadas as características das estações pluviométricas selecionadas.
A Figura 2 apresenta o mapa de Pernambuco com a delimitação e localização da área de estudo. Essa engloba a RMR, assim como o município de Goiana. Para a determinação desta área foram obtidos os arquivos shapefile dos municípios, agrupando-os e gerando uma única delimitação.

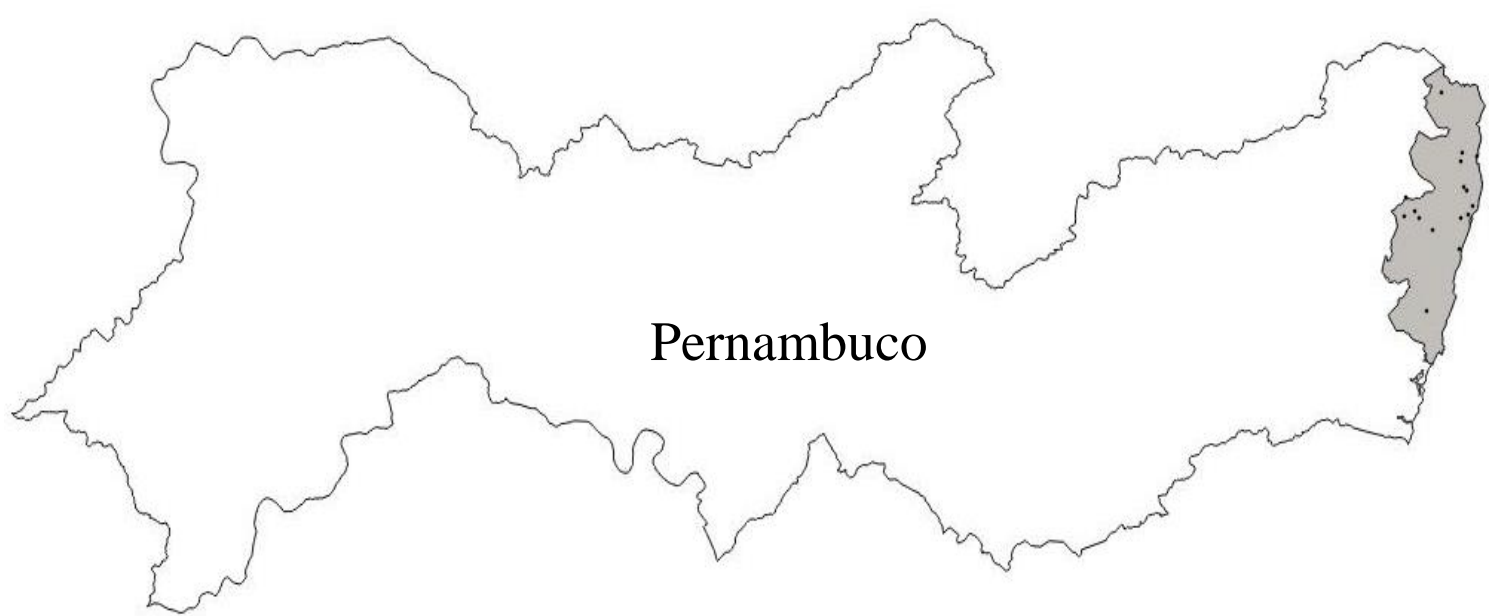

Figura 2 - Mapa de Pernambuco mostrando a delimitação da área de estudo. Fonte: Autor.

Através da Tabela 1 é possível verificar que a área de estudo compreende as seguintes Estações e Municípios: Caxangá e Várzea (Recife); Engenho Sítio, Engenho Bela Rosa, Nossa Senhora e Tapacurá (São Lourenço da Mata); Goiana (Goiana); Usina Ipojuca (Ipojuca); Jaboatão dos Guararapes, Barragem Duas Unas (Jaboatão dos Guararapes); Camaragibe (Camaragibe); Igarassu (Igarassu); Olinda (Olinda); Paulista (Paulista); Abreu e Lima (Abreu e Lima); Itamaracá (Itamaracá) e Itapissuma (Itapissuma).

Os Mapas da RMR e do Município de Goiana, indicando a localização pontual das 17 estações utilizadas como teste no presente estudo, estão apresentados na Figura 3.

Concluída a definição das estações a serem trabalhadas, deu-se início à etapa de determinação das relações i-d-f a partir dos registros pluviométricos. Essas determinações, segundo Cecílio e Pruski (2003), necessitam de um exaustivo trabalho de tabulação, análise e interpretação de grandes quantidades de dados. Utilizou-se a metodologia de desagregação de chuvas de Bell (1969) para determinação da i-d-f de cada posto pluviométrico selecionado.

O método de Bell (1969) é indicado, especificamente, para regiões onde as chuvas do tipo convectivas são predominantes, é válido para tempos de retorno variando de 2 a 100 anos e para durações de 5 a 120 min (Coutinho et al., 2013). Essa metodologia alternativa pode ser utilizada quando não se dispõe de dados pluviográficos, mas há disponibilidade de boas séries de dados pluviométricos, como é caso estudado.

Foram selecionadas para todas as localidades estudadas, as máximas precipitações diárias para cada ano, permitindo, assim, a preparação das séries anuais dos valores extremos. Para os tempos de retorno de 2, 5, 10, 25, 50 e 100 anos, empregando-se o método de Bell, foram determinadas as alturas pluviométricas com os dados de entrada para a obtenção dos parâmetros da i-d-f. Segundo Righetto (1998), o método de Bell associa a altura pluviométrica de uma chuva intensa de duração " $\mathrm{t}$ " e período de retorno " $\mathrm{T}$ ", isto é, h(dc, T), com uma chuva intensa padrão de 60 min de duração e 2 anos de tempo de retorno, $\mathrm{h}(60,2)$. Utiliza-se a relação empírica, descrita a seguir para os postos brasileiros:

$$
\begin{aligned}
& h(t, T)=(0,31 \cdot \ln T+0,7) \cdot\left(0,38 \cdot t^{0,31}-0,39\right) \cdot \\
& h(60,2)
\end{aligned}
$$

e para a determinação da $\mathrm{h}(60,2)$ :

$h(60,2)=0,510 \cdot h \operatorname{dia}(2)$

onde: hdia(2) a altura pluviométrica máxima diária anual correspondente ao período de retorno de 2 anos.

Foi utilizada a distribuição de Gumbel para a análise estatística, a qual tem tido grande aplicação para estudo de eventos extremos e a mais utilizada na análise de frequência de variáveis 
hidrológicas. Essa é utilizada de forma generalizada nos estudos de chuvas intensas, para a determinação de relações intensidade-duraçãofrequência.

A distribuição de Gumbel tem como função de densidade de probabilidade acumulada:

$$
F y(y)=\exp \left[-\exp \left(-\frac{y-\beta}{\alpha}\right)\right]
$$

$\beta$ - parâmetro de escala;

$\alpha$ - parâmetro de posição.

A sua inversa apresenta a seguinte forma:

$$
x(T)=\beta-\alpha \ln \left(-\ln \left(1-\frac{1}{T}\right)\right)
$$

onde: $\mathrm{T}$ - tempo de retorno em anos.

Estimando os parâmetros da distribuição pelo método dos momentos obtêm-se:

$$
\begin{aligned}
& \beta=\bar{X}-0,45 s_{x} \\
& \alpha=\frac{s_{x}}{1,283}
\end{aligned}
$$

onde: $\bar{X}$ e $\mathrm{s}_{\mathrm{x}}$ representam a média e o desvio padrão amostrais, respectivamente.

$\mathrm{O}$ teste de Kolmogorov-Smirnov (KS), com nível de significância de 5\%, o qual é não paramétrico e tem como base a diferença máxima entre as funções de probabilidades acumuladas, empírica e teórica, de variáveis aleatórias contínuas, foi usado para analisar a qualidade dos ajustes da distribuição de Gumbel. O teste é dado por:

$$
D_{N=} \sup _{-\infty<x<\infty} \mid F_{N(x)-F_{x}(x) \mid}
$$

correspondendo a maior diferença entre as probabilidades empírica e teórica.

Com todas as i-d-f's prontas, as calculadas e as existentes, com auxílio do software computacional livre QGIS 2.8.5 (Nanni et al., 2017), os parâmetros "a", "b", "c", "d" e ainda " $\alpha$ " e " $\beta$ " foram interpolados, um a um, utilizando a metodologia do inverso da distância ponderada IDW.

Tabela 1 - Características das estações pluviométricas selecionadas para a RMR. Fonte: Autor.

\begin{tabular}{|c|c|c|c|c|c|c|c|}
\hline Estação & Município & Responsável & Período & $\begin{array}{c}\text { Série } \\
\text { (anos) }\end{array}$ & COD & Latitude & Longitude \\
\hline Caxangá & Recife & DNOCS & $1926-1970$ & 44 & 834005 & $-8,0333$ & $-34,8833$ \\
\hline Várzea & Recife & ANA & $1994-2014$ & 20 & 30 & $-8,0500$ & $-34,9167$ \\
\hline Engenho Sítio & $\begin{array}{c}\text { São Lourenço } \\
\text { da Mata }\end{array}$ & ANA & $1967-2012$ & 44 & 735050 & $-7,9681$ & $-35,1567$ \\
\hline $\begin{array}{c}\text { Engenho Bela } \\
\text { Rosa }\end{array}$ & $\begin{array}{c}\text { São Lourenço } \\
\text { da Mata }\end{array}$ & ANA & $1971-1993$ & 22 & 363 & $-8,0167$ & $-35,1167$ \\
\hline Nossa Senhora & $\begin{array}{c}\text { São Lourenço } \\
\text { da Mata }\end{array}$ & ANA & $1970-1989$ & 19 & 405 & $-8,0500$ & $-35,1000$ \\
\hline Tapacurá & $\begin{array}{c}\text { São Lourenço } \\
\text { da Mata }\end{array}$ & ANA & $1994-2014$ & 20 & 267 & $-8,0403$ & $-35,1653$ \\
\hline Goiana & Goiana & INMET & $1925-1967$ & 43 & 735052 & $-7,5667$ & $-35,0000$ \\
\hline Usina Ipojuca & Ipojuca & IAA & $1963-1978$ & 16 & 835060 & $-8,4000$ & $-35,0667$ \\
\hline $\begin{array}{c}\text { Jaboatão dos } \\
\text { Guararapes }\end{array}$ & $\begin{array}{c}\text { Jaboatão dos } \\
\text { Guararapes }\end{array}$ & ANA & $1995-2014$ & 19 & 202 & $-8,1636$ & $-34,9228$ \\
\hline $\begin{array}{c}\text { Barragem Duas } \\
\text { Unas }\end{array}$ & $\begin{array}{c}\text { Jaboatão dos } \\
\text { Guararapes }\end{array}$ & ANA & $1998-2014$ & 16 & 268 & $-8,0922$ & $-35,0386$ \\
\hline Camaragibe & Camaragibe & ANA & $1998-2014$ & 16 & 201 & $-8,0217$ & $-34,9922$ \\
\hline Igarassu & Igarassu & ANA & $1997-2014$ & 17 & 100 & $-7,8281$ & $-34,9150$ \\
\hline Olinda & Olinda & ANA & $1995-2014$ & 20 & 199 & $-7,9989$ & $-34,8589$ \\
\hline Paulista & Paulista & ANA & $2000-2014$ & 14 & 451 & $-7,9425$ & $-34,8844$ \\
\hline Abreu e Lima & Abreu e Lima & ANA & $1999-2014$ & 15 & 198 & $-7,9281$ & $-34,9000$ \\
\hline Itamaracá & Itamaracá & ANA & $2002-2014$ & 12 & 524 & $-7,8086$ & $-34,8378$ \\
\hline Itapissuma & Itapissuma & ANA & $2002-2014$ & 12 & 520 & $-7,8003$ & $-34,9058$ \\
\hline
\end{tabular}




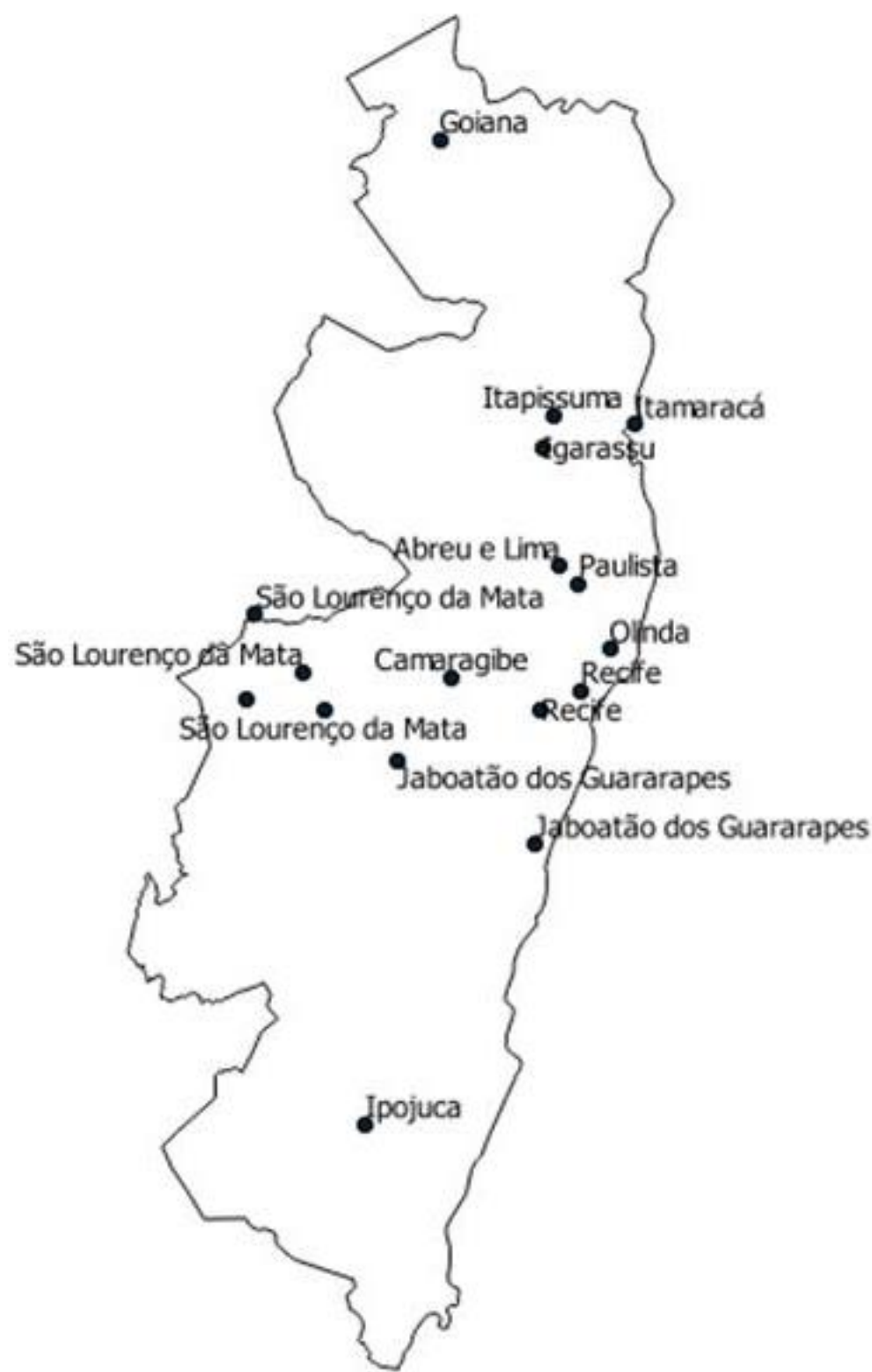

Figura 3 - Mapa da Região Metropolitana de Recife (RMR) e Município de Goiana mostrando a localização das estações pluviométricas. Fonte: Autor.

O IDW é considerado o método mais simples de interpolação, que atribui o peso maior ao ponto mais próximo, diminuindo esse peso com o aumento da distância, ou seja, quanto maior for o peso, menor será a influência dos pontos mais distantes do ponto notável. Os pesos dos locais observados, a serem usados na predição, são ponderados, e sua soma é igual a 1 .

De acordo com Marcuzzo et al. (2011), o método do inverso da distância é utilizado para estimar os parâmetros de chuvas intensas através de uma média ponderada dos parâmetros da equação de chuvas intensas, para locais desprovidos de dados pluviométricos. Utilizam-se dados de estações adjacentes. Como produto, ocorre a conversão dos dados de observações pontuais para campos contínuos, espacializando o valor do parâmetro através de transformação das imagens vectoriais em rasters.

No QGIS, os dados de entrada foram os arquivos shapefiles de pontos das estações pluviométricas da RMR e os dados dos parâmetros de i-d-f e Gumbel, ambos georreferenciados no sistema UTM Sirgas 2000. Como não há uma regra estabelecida para definir o tamanho do pixel de saída para o modelo, quanto menor o valor do pixel, melhor a resolução da imagem de saída, utilizou-se para este estudo o valor de $30 \mathrm{~m}$. Foram gerados, no total, 12 mapas de espacialização dos parâmetros supracitados, onde seis são relativos a interpolações entre as 17 estações pluviométricas e os outros seis a mapas que foram gerados após a remoção de uma das estações. 
A remoção de um posto foi realizada para verificação posterior, através do cálculo do erro percentual, de intensidades de chuvas entres os valores obtidos da equação i-d-f determinada, com o valor obtido após as interpolações. Para o cálculo do erro percentual considerou-se um período de retorno de 10 anos e duração de 10 min para obtenção das intensidades de chuvas, utilizando-se a seguinte equação:

$$
\mathrm{E}(\%)=\frac{100 *\left(i_{m c a l}-i_{m i n t}\right)}{i_{m c a l}}
$$

onde: $\mathrm{E}$ - o erro percentual entre os valores de $i_{m}$; $\mathrm{i}_{\mathrm{m} \text { cal }}$ - intensidade calculada com os valores reais de "a", "b", "c", "d"; $i_{\mathrm{m} \text { int }}$ - intensidade calculada com os valores interpolados de "a", "b", "c", "d".

\section{Resultados e discussão}

Todas as 17 equações de intensidadeduração-frequência calculadas para utilização neste trabalho mostraram-se adequadas, segundo a distribuição de Gumbel, no que se refere à estimativa da precipitação máxima avaliada pelo teste de Kolmogorov-Smirnov para o nível de significância de $5 \%$ de probabilidade. Os parâmetros estatísticos para distribuição de Gumbel, " $\alpha$ " e " $\beta$ ", bem como os valores encontrados dos coeficientes "a", "b", "c" e "d" da equação i-d-f determinadas pelo método de Bell são apresentados na Tabela 2.

Referente à espacialização dos parâmetros, a partir dos valores pontuais dos parâmetros calculados para as estações pluviométricas, foi utilizado o método de interpolação espacial IDW para estimar continuamente os valores dos parâmetros das equações de chuvas intensas para qualquer ponto da RMR e município de Goiana. A Figura 4 apresenta os mapas com os resultados das interpolações matemáticas, utilizando o método do IDW para cada parâmetro separadamente.

Tabela 2 - Parâmetros estatísticos para distribuição de Gumbel, " $\alpha$ " e " $\beta$ ”, e dos coeficientes "a", "b", "c" e "d" da equação i-d-f, determinados pelo método de Bell. Fonte: Autor.

\begin{tabular}{|c|c|c|c|c|c|c|}
\hline Estação & $\mathbf{a}$ & b & c & d & $\alpha$ & $\beta$ \\
\hline Caxangá & 362,7199 & 0,2129 & 4,4122 & 0,5824 & 34,5800 & 69,9200 \\
\hline Várzea & 484,9700 & 0,2129 & 2,8307 & 0,5581 & 22,8600 & 104,5200 \\
\hline Engenho Sítio ${ }^{1}$ & 304,2810 & 0,2129 & 2,8307 & 0,5580 & 60,3400 & 54,9100 \\
\hline Engenho bela rosa & 356,5100 & 0,2129 & 2,8304 & 0,5580 & 21,3800 & 81,3600 \\
\hline Nossa Senhora & 371,1900 & 0,2129 & 2,8307 & 0,5580 & 25,7500 & 83,2900 \\
\hline Tapacurá & 330,8000 & 0,2129 & 2,8309 & 0,5581 & 25,2900 & 69,3600 \\
\hline Goiana ${ }^{1}$ & 491,1510 & 0,1987 & 2,8231 & 0,5573 & 42,1200 & 100,2000 \\
\hline Usina Ipojuca ${ }^{1}$ & 707,8600 & 0,2129 & 2,8303 & 0,5581 & 35,9000 & 76,7000 \\
\hline Jaboatão dos Guararapes & 384,2525 & 0,2129 & 2,8307 & 0,5581 & 24,3100 & 83,4200 \\
\hline Barragem Duas Unas & 389,9600 & 0,2129 & 2,8308 & 0,5581 & 21,7900 & 82,4500 \\
\hline Camaragibe & 391,6400 & 0,2129 & 2,8307 & 0,5581 & 34,1900 & 87,9200 \\
\hline Igarassu & 381,8430 & 0,2129 & 2,8309 & 0,5581 & 20,0100 & 91,4600 \\
\hline Olinda & 400,14 & 0,2129 & 2,83083 & 0,5581 & 27,02 & 96,77 \\
\hline Paulista & 415,64 & 0,2129 & 2,83072 & 0,5581 & 20,35 & 99,89 \\
\hline Abreu e Lima & 432,39 & 0,2129 & 2,83085 & 0,5581 & 24,67 & 92,15 \\
\hline Itamaracá & 402,61 & 0,2127 & 2,83081 & 0,5581 & 21,64 & 88,42 \\
\hline Itapissuma & 460,32 & 0,2126 & 2,8308 & 0,5581 & 21,25 & 98,9 \\
\hline
\end{tabular}

${ }^{1}$ Dados retirados de Coutinho et al. (2013).

De modo geral, pode-se perceber que a interpolação dos parâmetros "a" e "b" apresentaram-se bastante uniformes na região de maior concentração de postos pluviométricos. Cada parâmetro apresentou uma "ilha" de valor mais discrepante localizado nas proximidades do posto de Ipojuca e de Goiana, respectivamente, para "a" e "b". Enquanto o parâmetro "a" é o que possui maior abrangência de valores, variando entre cerca de 300 e 700, o parâmetro "b", é o que possui menores magnitudes e variação, com valores variando entre 0,1987 e 0,2129 . Assim, os locais mais próximos à concentração das estações tendem a ter, por interpolação, o parâmetro analisado com valores minimamente influenciados pelas estações Ipojuca ("a") e Goiana ("b"), devido a sua distância e discrepância de valor em relação aos demais postos.

Os parâmetros "c" e "d" apresentaram mapas de interpolação bastante semelhantes 
visualmente, com grande uniformidade espacial do parâmetro em questão, isto é, com praticamente um único intervalo de valor em todo o mapa. Como pode ser visto, em ambos os mapas há uma única estação, Recife (Caxangá), com valores bem maiores que os demais interpolados. Mesmo assim, a influência deste ponto nos mapas é pouco relevante, visto a alta densidade de postos aos arredores de valores inferiores. Aragão et al. (2012) encontraram resultados semelhantes no tocante a uniformidade dos parâmetros para estações pluviométricas do litoral de Sergipe.

Quanto à interpolação dos parâmetros estatísticos da distribuição de Gumbel " $\alpha$ " e " $\beta$ ”, os mapas são, aproximadamente, semelhantes, com as cores de tendência invertidas. Isto é, onde aparece vermelho na interpolação de " $\alpha$ " é azul na interpolação de " $\beta$ ", e vice-versa. Essa relação ocorre devido a ambos os parâmetros dependerem dos mesmos coeficientes estatísticos, média e desvio padrão da série histórica da estação pluviométrica a que se refere. Os valores dos parâmetros variam gradualmente nos dois mapas, com poucos extremos, destacando-se apenas a estação de São Lourenço da Mata no extremo Oeste.

Com o intuito de verificar a consistência das interpolações dos parâmetros, foi removida uma estação pluviométrica, cujos parâmetros eram conhecidos (calculados pelo método de Bell) e gerados novamente os seis mapas de interpolação, apresentados na Figura 5. A estação removida foi a do município de Camaragibe (Lat. -8,0217, Long. -34,9922). A partir disto, cada mapa gerado forneceu o respectivo parâmetro interpolado, sendo possível calcular o erro percentual entre os parâmetros calculados e interpolados. Foi calculado, também, o erro percentual para as intensidades de chuva utilizando as i-d-f's, para a mesma estação, com os parâmetros calculados por Bell e interpolados por IDW, utilizando-se o tempo de retorno de 10 anos e duração da chuva de 10 min conforme o usualmente utilizado em projetos de dimensionamento de microdrenagem urbana. Os resultados dos erros percentuais são apresentados na Tabela 3.

É possível observar que o erro percentual foi pequeno para todos os parâmetros das equações de chuvas intensas, com valores variando entre $0,05 \%$ e $5,43 \%$. O parâmetro estatístico " $\alpha$ ", apesar de apresentar $24,23 \%$ de erro percentual, não invalida o estudo, visto que este não é utilizado diretamente na determinação das chuvas intensas quando o método de Bell é aplicado. Portanto, para a estação de Camaragibe, a interpolação dos parâmetros da equação de chuvas intensas pode ser utilizada sem maiores impedimentos.

Para o cálculo da intensidade, em $\mathrm{mm} / \mathrm{h}, \mathrm{o}$ baixo erro percentual de $1,80 \%$ ratifica a recomendação de utilizar os parâmetros interpolados para a espacialização das i-d-f em locais desprovidos dessa informação, onde, através do método quadrado do inverso da distância, valores menores que 5\% também foram encontrados por Melo et al. (2003) para o estado de São Paulo. Além disso, o valor negativo mostra a tendência de superestimativa da intensidade máxima média de precipitação calculada, o que significa trabalhar com uma margem de segurança adicional no projeto das obras de engenharia.

Tabela 3 - Erros percentuais para os parâmetros das equações de chuvas intensas para a Estação de Camaragibe.

\begin{tabular}{|c|c|c|c|}
\hline Parâmetro & IDF (calculada) & IDF (Interpolada) & E (\%) \\
\hline $\mathrm{a}$ & 391,6400 & 403,8041 & $-3,11 \%$ \\
\hline $\mathrm{b}$ & 0,2129 & 0,2128 & $0,05 \%$ \\
\hline $\mathrm{c}$ & 2,8307 & 2,9843 & $-5,43 \%$ \\
\hline $\mathrm{d}$ & 0,5581 & 0,5604 & $-0,41 \%$ \\
\hline$\alpha$ & 34,1900 & 25,9066 & $24,23 \%$ \\
\hline$\beta$ & 87,9200 & 87,0832 & $0,95 \%$ \\
\hline $\mathrm{i}_{\mathrm{m}}(\mathrm{mm} / \mathrm{h})$ & 153,9120 & 156,6791 & $-1,80 \%$ \\
\hline
\end{tabular}


Revista Brasileira de Geografia Física, Vol. 11, N. 04 (2017) 1542-1554

a)

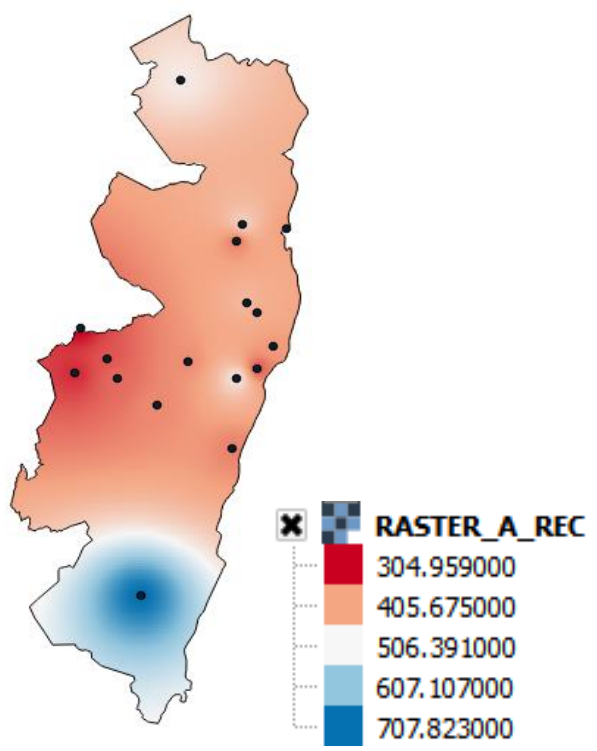

c)

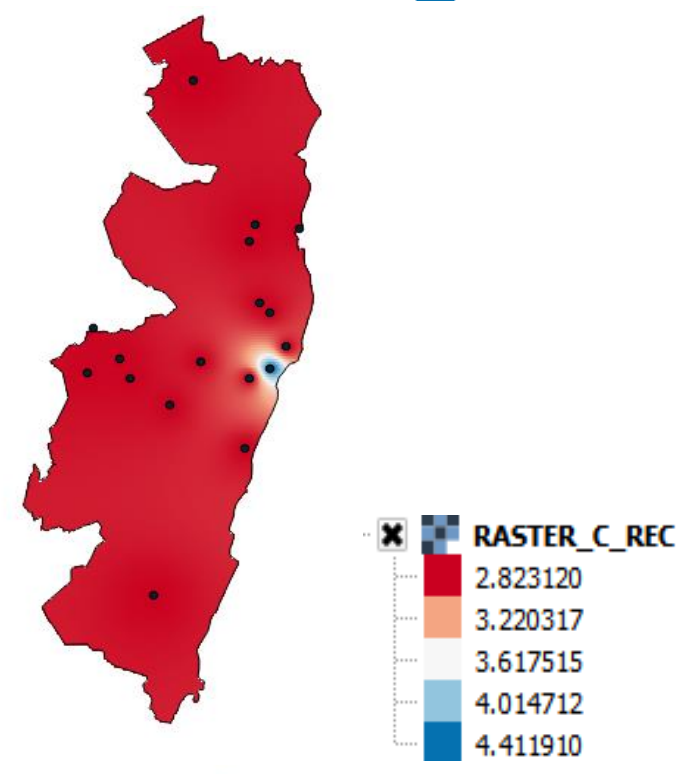

e)

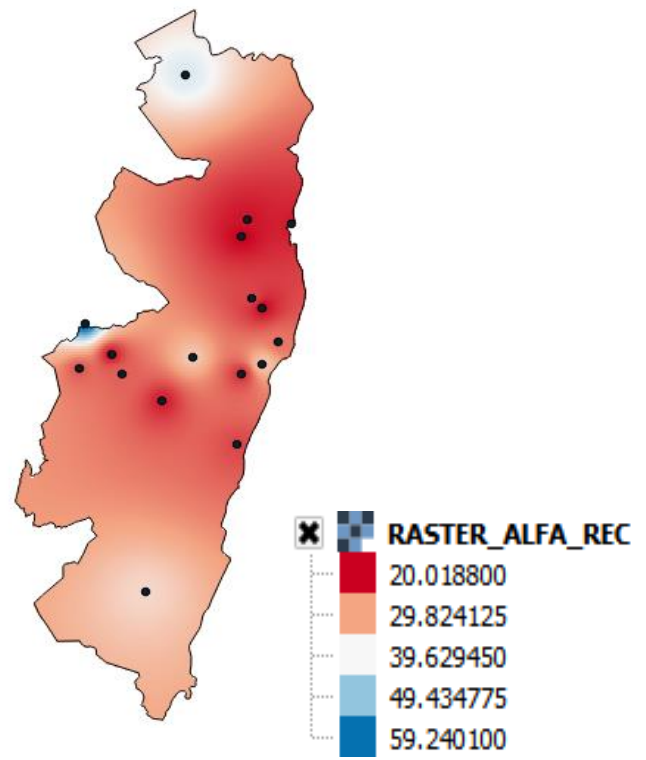

b)

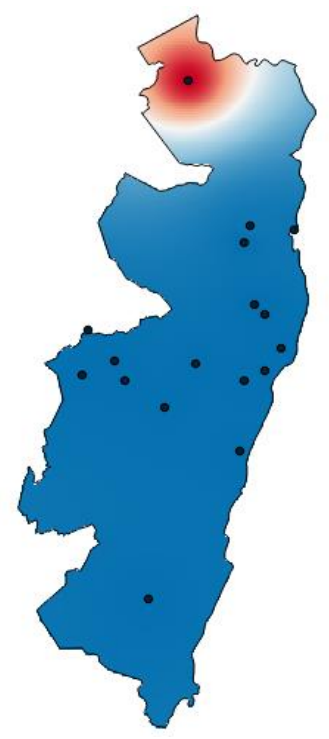

X

0.198702

0.202252

0.205801

0.209351

0.212900

d)

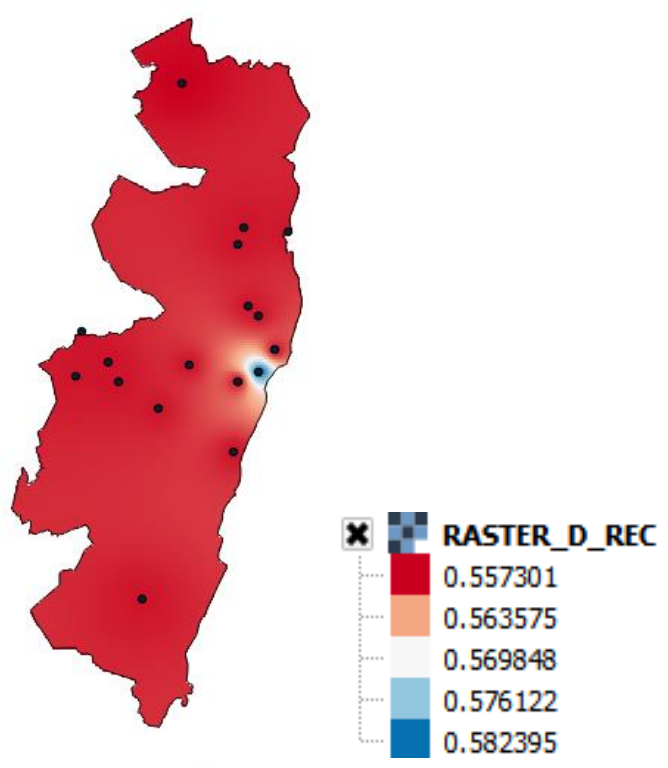

f)

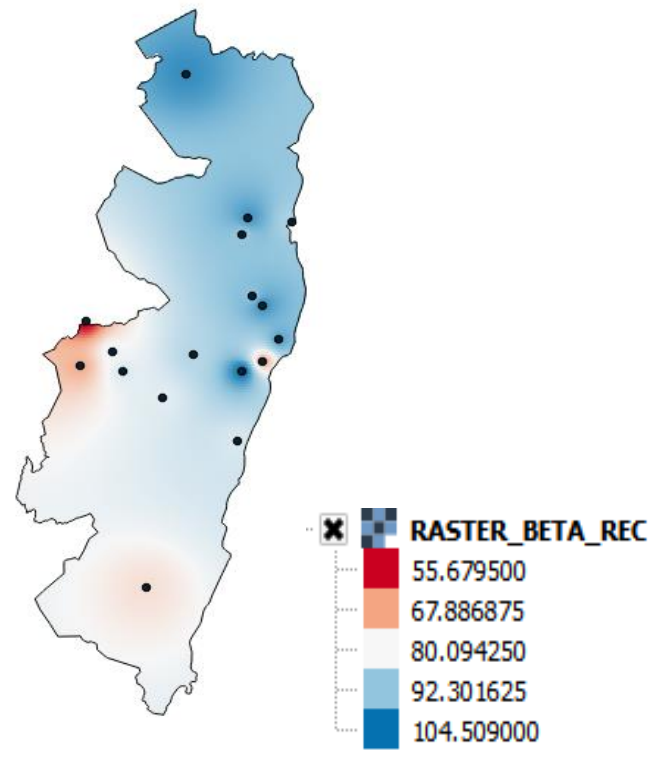

Figura 4 - Interpolação dos parâmetros de chuvas intensas para as 17 Estações. a) Interpolação parâmetro "a"; b) Interpolação parâmetro "b"; c) Interpolação parâmetro "c"; d) Interpolação parâmetro "d"; e) Interpolação parâmetro " $\alpha$ "; f) Interpolação parâmetro " $\beta "$. 
Revista Brasileira de Geografia Física, Vol. 11, N. 04 (2017) 1542-1554

a)

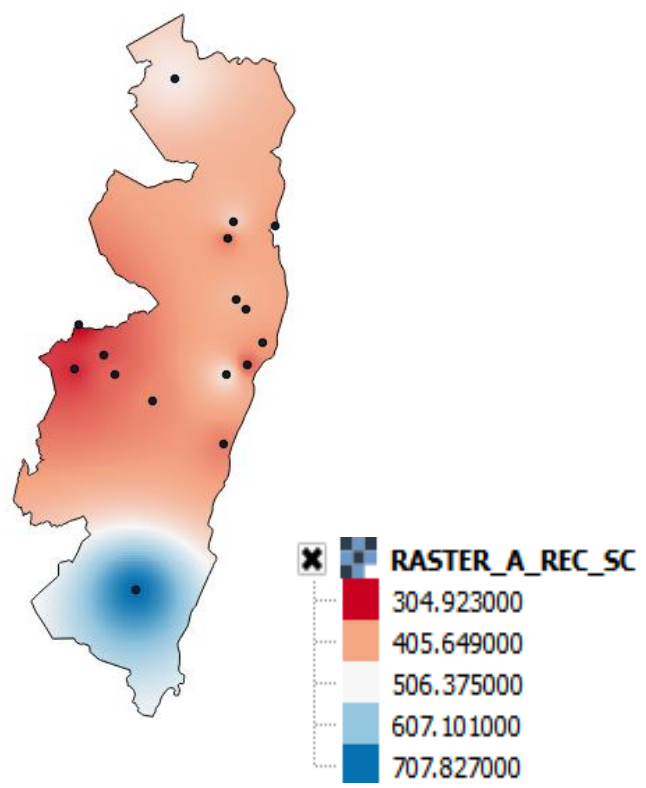

c)
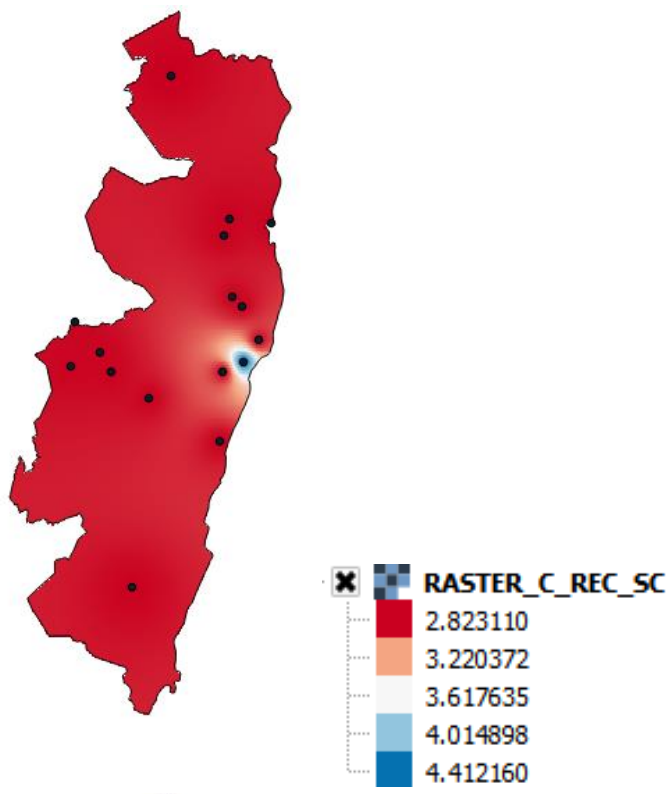

e)

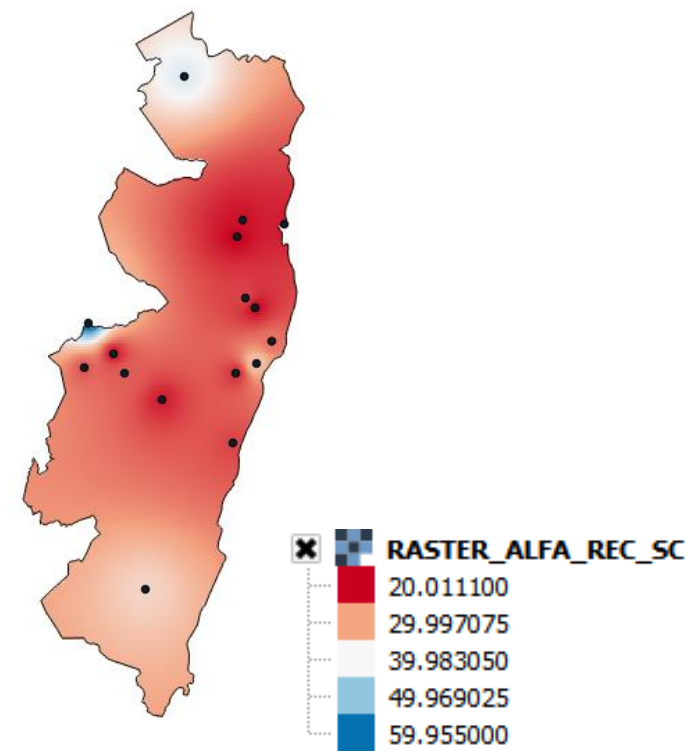

b)
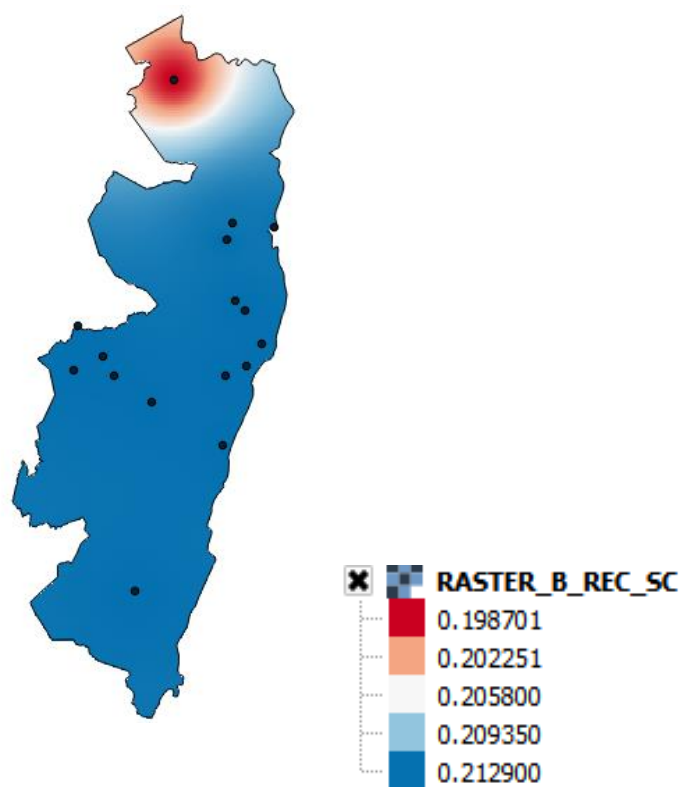

d)

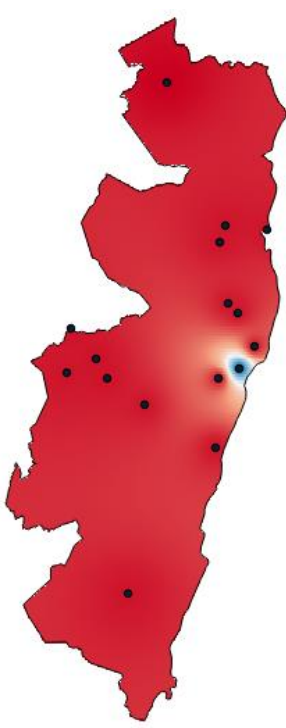

f)

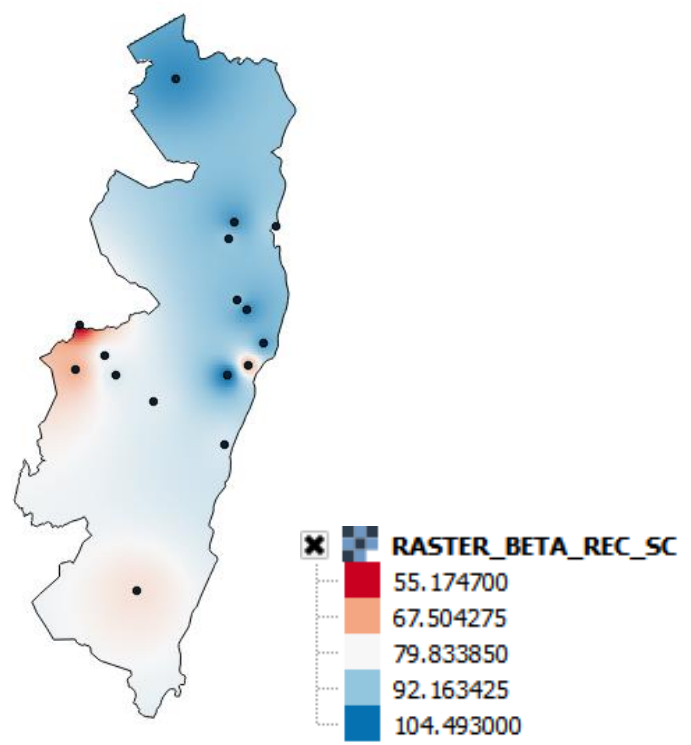

Figura 5 - Interpolação dos parâmetros de chuvas intensas para as Estações, sem considerar Camaragibe. a) Interpolação parâmetro "a"; b) Interpolação parâmetro "b"; c) Interpolação parâmetro "c"; d) Interpolação parâmetro "d"; e) Interpolação parâmetro " $\alpha$ "; f) Interpolação parâmetro " $\beta$ ". 


\section{Conclusões}

Para todas as localidades estudadas, a distribuição de Gumbel mostrou-se adequada para representar as estimativas dos valores das precipitações máximas no nível de significância de 5\% pelo teste de Kolmogorov-Smirnov.

A Interpolação forneceu apenas uma estimativa de um valor baseado em outros valores pré conhecidos de estações pontuais. Essa interpolação foi bem-sucedida, visto que os erros percentuais são baixos, variando de 0,05 até $5,43 \%$, quando feita a análise para a Estação de Camaragibe.

No entanto, este método assume que a superfície possui uma variação local, e funciona melhor se os pontos amostrais estiverem igualmente distribuídos pela área, sem estarem concentrados em determinado local.

Quanto aos parâmetros das equações idf, os valores de "c" e "d" foram bastante semelhantes para todas as estações interpoladas devido à dependência desses com o método de Bell e a homogeneidade dos padrões de chuvas intensas na escala da RMR.

Os mapas gerados através do geoprocessamento com as técnicas de interpolação IDW permitem uma melhor interpretação, facilitando a discussão e permitindo a geração de novas informações de forma rápida e precisa.

\section{Agradecimentos}

Os autores agradecem a Fundação de Amparo à Ciência e Tecnologia do Estado de Pernambuco- FACEPE pela concessão de bolsa de mestrado para a primeira autora.

\section{Referências}

Albuquerque, R. D., Dantas, C. E. O., Araújo, E. L., Vasconcelos, T. L., 2013. Distribuição Temporal das Precipitações no Município do Recife (Temporal Distribution of Rainfall in the Recife City). Revista Brasileira de Geografia Física 2, 245-252.

ANA., 2015. Agência Nacional das Águas. Hidroweb: Sistema de informações hidrológicas. http://hidroweb.ana.gov.br. Acesso: 03 dezembro 2015.

Back, A. J., 2011. Seleção de distribuição de probabilidade para chuvas diárias extremas do Estado de Santa Catarina. Revista Brasileira de Meteorologia 2, p. 211-222.

Bell, F. C., 1969. Generalized rainfall-durationfrequency relationships. Journal of the Hydraulics Division-ASCE 95, 311-327.
Cecilio, R. A., Pruski, F. F., 2003. Interpolação dos parâmetros da equação de chuvas intensas com uso do inverso de potências da distância. Revista Brasileira de Engenharia Agrícola e Ambiental 3, 501-504.

Cecílio, R. A., Xavier, A. C., Pruski, F. F., Hollanda, M. P., Pezzopane, J. E. M., 2009. Avaliação de interpoladores para os parâmetros das equações de chuvas intensas no Espírito Santo. Ambi-Água, Taubaté 3, 82-92. (doi:10.4136/ambi-agua.104).

Coutinho, A. P., Leite, L. L. L., Ribas, L. V.; Antonino, C. D. A., Cabral, J. J. S. P., Montenegro, S. M. G. L., 2013a. Coletânea de equações de chuvas intensas para o Estado de Pernambuco. Anais do XX Simpósio Brasileiro de Recursos Hídricos. Bento Gonçalves/RS.

Coutinho, A. P., Leite, L. L. L., Ribas, L. V.; Antonino, C. D. A., Cabral, J. J. S. P.; Montenegro, S. M. G. L., Melo, T. A. T., 2013b. Determinação de equações de chuvas intensas para municípios das mesorregiões do Estado de Pernambuco através do método de Bell. Anais do XX Simpósio Brasileiro de Recursos Hídricos. Bento Gonçalves/RS.

Coutinho, A. P., Lassabatere, L., Montenegro, S., Antonino, A. C. D., Angulo-Jaramillo, R., Cabral, J. J. S. P., 2016. Hydraulic characterization and hydrological behaviour of a pilot permeable pavement in an urban centre, Brazil. Hydrol. Process 30, 4242-4254. doi: 10.1002/hyp.10985.

Silva, S. R., Araújo, G. R. S., 2013. Algoritmo para Determinação da Equação de Chuvas Intensas (Algorithm to Determine the Equation of Intense Rain). Revista Brasileira de Geografia Física 5, 1371-1383.

Aragão, R. de, Santana, G. R. de, Costa, C. E. da, Cruz, M. A., Figueiredo, E. E. de, Srinivasan, V. S., 2013. Chuvas intensas para o estado de Sergipe com base em dados desagregados de chuva diária. R. Bras. Eng. Agríc. Ambiental 3, 243-252.

Oliveira, L. F. C. de, Cortês, F. C., Wehr, T. R., Borges, L. B., Sarmento, P. H. L., Griebeler, N. P., 2007. Intensidade-duração-freqüência de chuvas intensas para localidades no estado de goiás e distrito federal. Pesquisa Agropecuária Tropical (Agricultural Research in the Tropics) 1, 13-18.

Oliveira, L. F. C. de, Antonini, J. C. D. A., Griebeler, N. P., 2008. Estimativas de chuvas intensas para o Estado de Goiás. Engenharia Agrícola 1, 22-23. 
Garcia, S. S., Amorim, R. S., Couto, E. G., Stopa, W. H., 2011. Determinação da equação intensidade-duração-frequência para três estações meteorológicas do Estado de Mato Grosso. Revista Brasileira de Engenharia Agrícola e Ambiental 15, 575-581.

Marcuzzo, F. F. N.; Andrade, L. R.; Melo, D. C. R., 2011. Métodos de interpolação matemática no mapeamento de chuvas do Estado do Mato Grosso. Revista Brasileira de Geografia Física., 4, 793-804.

Mello, C. R., Lima, J. M., Silva, A. M., Mello, J. M., Oliveira, M. S., 2003. Krigagem e inverso do quadrado da distância para interpolação dos parâmetros da equação de chuvas intensas. R. Bras. Ci. Solo 27, 925-933.

Nanni, A. S., Descovi Filho, L., Virtuoso, M. A., Montenegro, D., Willrich, G., Machado, P. H.,
Sperb, R., Dantas, G. S., Calazans, Y., 2012. Quantum GIS - Guia do Usuário, Versão 1.7.4 'Wroclaw'. 291p., il. Disponível em: http://qgisbrasil.org. Acesso em: 10/02/2017.

Silva, B. M., Montenegro, S. M. M. G., Silva, F. B. da, Araújo Filho, P. F., 2012. Chuvas Intensas em Localidades do Estado de Pernambuco. RBRH - Revista Brasileira de Recursos Hídricos 3, Jul/Set 2012, 135-147.

Taborga, J. J. T., 1974. Práticas Hidrológicas. Rio de Janeiro. TRANSCON, 120 pg. Cetesb; Ascetesb. Drenagem urbana: manual de projeto.

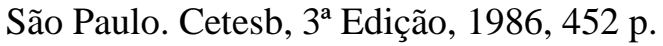

Tao, T. A. O., Chocat, B., Liu, S., Xin, K., 2009. Uncertainty analysis of interpolation methods in rainfall spatial distribution-a case of small catchment in Lyon. Journal of Environmental Protection 01, 50-58. 